\title{
Inverted Gate Vedic Multiplier in 90nm CMOS Technology
}

\section{Chiranjit Rajendra Patel, Vivek Bettadapura Adishesha, Vivek Urankar, Keshav Vaidyanathan Bharadwaj}

Electronics and Communication, RNS Institute of Technology, Bengaluru, Karnataka, India

\section{Email address:}

chiranjitpatel08@gmail.com (C. R. Patel), vivek.adishesha@gmail.com (V. B. Adishesha), vivekurankar@gmail.com (V. Urankar), keshavbharadwaj98@gmail.com (K. V. Bharadwaj)

\section{To cite this article:}

Chiranjit Rajendra Patel, Vivek Bettadapura Adishesha, Vivek Urankar, Keshav Vaidyanathan Bharadwaj. Inverted Gate Vedic Multiplier in 90nm CMOS Technology. American Journal of Electrical and Computer Engineering. Vol. 4, No. 1, 2020, pp. 10-15.

doi: 10.11648/j.ajece.20200401.12

Received: June 16, 2020; Accepted: June 30, 2020; Published: July 7, 2020

\begin{abstract}
This paper proposes the design and implementation of an enhanced binary multiplication technique. Vedic Mathematics is a system of mathematics that was discovered by Indian mathematician Jagadguru Shri Bharathi Krishna Tirthalji in the period between 1911 and 1918. The main objective of this paper is to design an improved binary multiplier which is faster and low-powered. The performance of our proposed full adder design is proven to be more effective in comparison with the standard full adder cell both designed in $90 \mathrm{~nm}$. The proposed modified 2-Bit and 4-Bit Vedic multipliers also beat the existing Vedic multiplier based in Urdhva Tiryagbhyam sutra in terms of operating frequency, energy and area. ThedesignsareimplementedoncadenceVirtuoso90nmCMOStechnology operating at 2V supply. Comparedtotheexisting standard fulladderdesigns in $90 \mathrm{~nm}$, the proposed implementation has shown that it offers significant improvements in terms of power and speed consuming $60 \%$ less power and is able to operate $20 \%$ faster. The proposed 2-Bit multiplier operated at $2 \mathrm{~V}$ is proven to be more effective. The design was further extended to realise a 4-Bit multiplier. The power consumed by the standard 4- Bit multiplier designed using standard $90 \mathrm{~nm}$ cells was $361.2 \mu \mathrm{W}$ and the power consumed by the proposed 4-Bit multiplier design was found to be $290.2 \mu \mathrm{W}$, which reflectsa $20 \%$ decrease in the power usage.
\end{abstract}

Keywords: CMOS 90nm, Inverted Gate, Vedic Multiplier, Urdhva Tiryagbhayam, Carry Save Adder, Layout Design

\section{Introduction}

With the advent of VLSI technology and the exponential growth in the number of transistors on the chip, there is a need for newer architectures to be faster, and at the same time, power consumption to be at its minimum.

This paper presents a way of modifying the existing design of the Vedic multiplier to suit for various applications. The modified Full adder and 2-Bit Vedic multiplier are proved to be much more efficient when compared to their conventional standard designs. The proposed design of the 4-Bit multi- plier using these modified designs helps improve the overall performance of the system.

Discrete Cosine Transformation (DCT) plays a crucial role for compression of images, the works of [3] propose a custom multiplication algorithm for reducing the complexity of matrix multiplication. Vedic multiplications techniques are proven to be more effective than conventional methods [13], which is the main driving factor for our study into Vedic mathematics. Various multipliers and dividers based on Vedic sutras addressed in [6] and the authors conclude that the use of these sutras in the computing algorithm of the digital system would reduce the complexity of design, area, execution time and power consumption. Karatsuba al-gorithm is preferred for higher bit multiplication and numer- ous implementations of higher bit multipliers on Xilinx tool have been performed [4, 7, 8]. Complexity of the circuits can be reduced by using techniques like Gate Diffusion In- put (GDI) as studied in [11].

\section{Multiplication Technique}

Urdhva Triyagbhyam

The word "Urdhva Triyagbhayam" resources "vertically" and "crosswise" multiplication [1]. It is a nonspecific algo-rithm for $\mathrm{N}$-Bit numbers. The advantage of this algorithm is that delay and area increases slowly as the number of binary bits for multiplication increase. These circuits show 
high regularity and hence the low layout area.

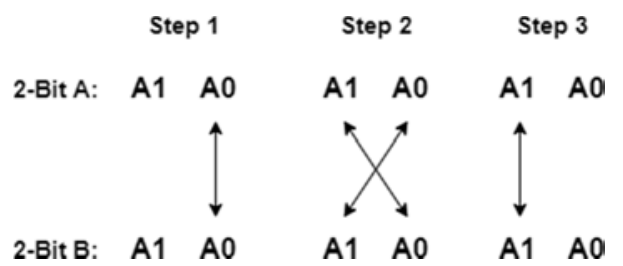

Figure 1. 2-Bit binary multiplication using Urdhva Triygb-hyam Sutra.

Figure 1 shows two numbers (A1A0 and B1B0) each of 2-Bit that are inputs. The results in a 4-Bit number.

\section{Proposed Full Adder Design}

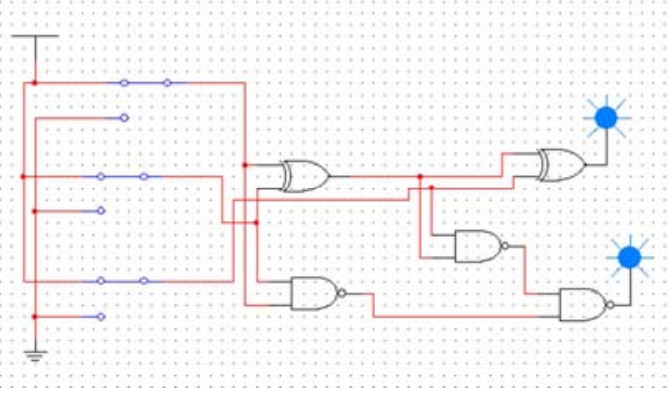

Figure 2. Proposed Full adder design.

Conventional full adders use AND and OR gates for thee generation of carry, whereas the proposed full adder logic eliminates the use of AND and OR gates for the carry bit generation. The NAND gates are used instead. NAND gate has equal fall and rise times. But a NOR gate has two pMOS connected in series. This requires increase in sizes of transistors in NOR to have good fall and rise times.

The use of NAND gates instead of OR and AND gives the advantage of reducing the delay, power consumed and area occupied by the circuit. Since NAND has a better ration of output high drive and output low drive as compared to NOR. NAND is preferred over NOR [2].

Basic gates are designed using the aspect ratio 1.6.

\section{Modified Vedic Multiplier}

\subsection{2-Bit Multiplier}

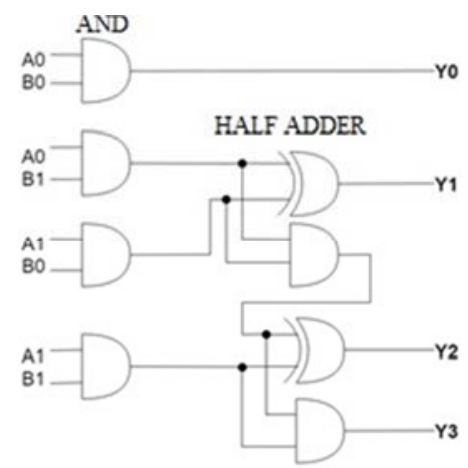

Figure 3. Vedic 2-Bit multiplier based on UT Sutra.
In Figure 3 binary numbers $\mathrm{A} 1 \mathrm{~A} 0$ and $\mathrm{B} 1 \mathrm{~B} 0$ are considered. In the first step, $\mathrm{Y} 1$ is obtained by multiplying the bits $\mathrm{A} 0$ and $\mathrm{B} 0$. The second step involves adding the products $\mathrm{A} 0 \mathrm{~B} 1$ and A1B0. The summation of these, results as $\mathrm{Y} 1$ and the carry is propagated to the next stage. Addition is per-formed using a standard half adder.

In the proposed design as shown in Figure 4 the use of the standard half adder is eliminated and the circuit is mod- ified which helps reduces the delay and power consumption. The design is tested and verified using Multisim and Cadence Virtuoso 90nm shown in Figure 5 and Figure 6.

\subsection{4-Bit Multiplier}

The $4 \times 4$ multiplication is done considering the grouping 2 bits together each of the 4-Bit input, Figure 7. Each group of 2 bits of each input is handled by a separate 2-Bit multiplier.

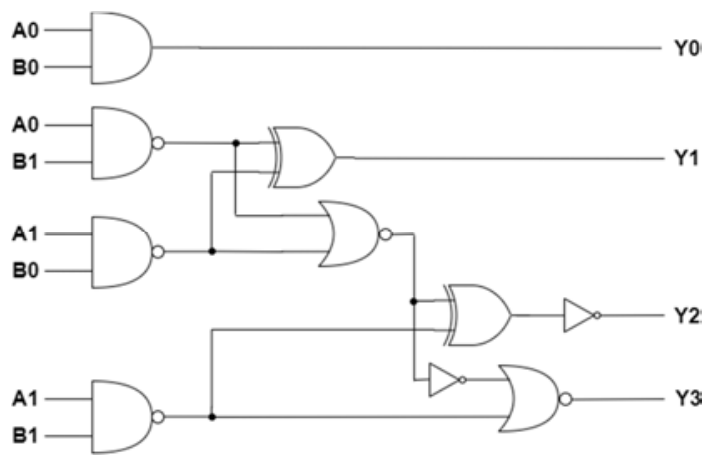

Figure 4. Modified Vedic 2-Bit multiplier based on UT Sutra.

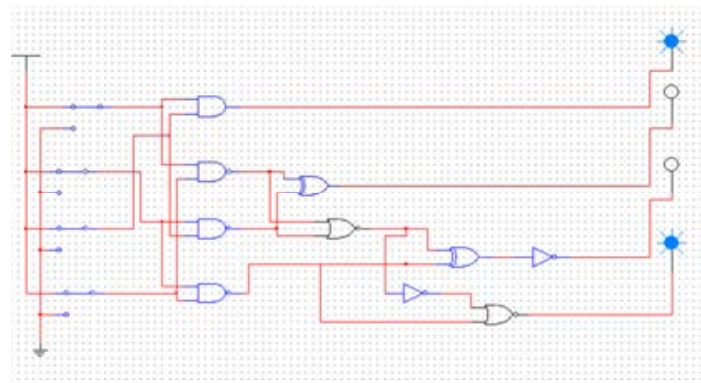

Figure 5. Modified 2-Bit multiplier implemented on Multi-sim.

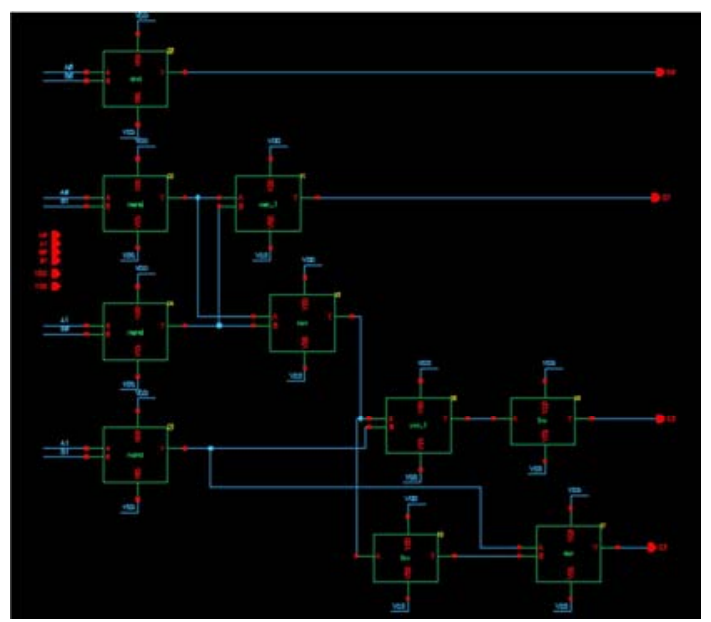

Figure 6. Modified 2-Bit multiplier schematic. 
The vertically and crosswise operation is performed for a total of four times which requires four 2-Bit multipliers. The partial products produced by each of 2-Bit multipliers are added in a Carry Save Adder.

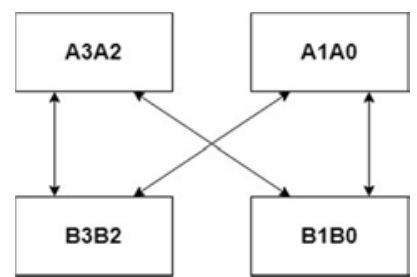

Figure 7. 4-Bit binary multiplication using Urdhva Triygb-hyam Sutra.

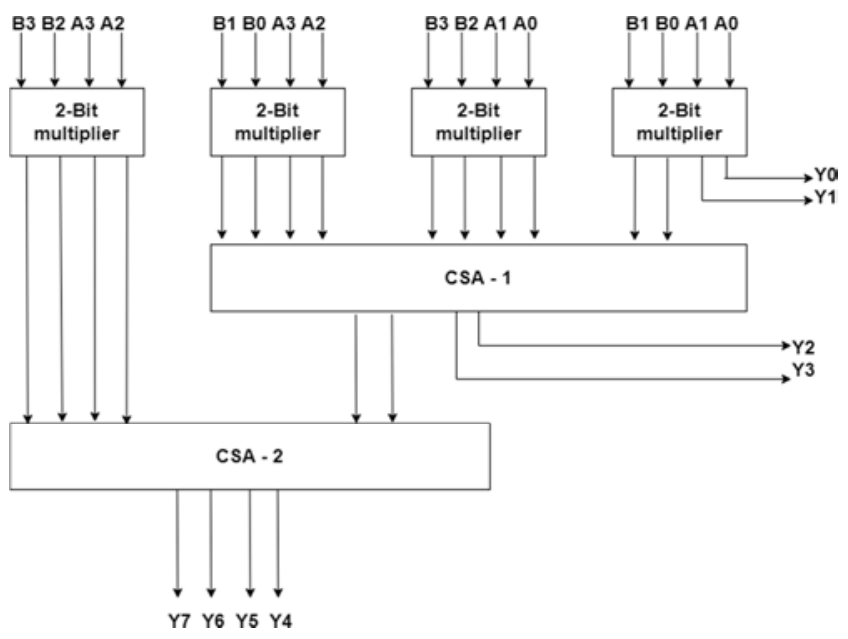

Figure 8. 4-Bit multiplier block diagram.

\subsection{Carry Save Adder (CSA)}

Carry Save Adder is used to add three numbers at an instant. Using this property of CSA, eliminates the need for a third adder in the 4-Bit multiplier design. The use of two adders instead of three decreases the delay, power dissipa- tion and area consumed. CSA has the advantage of using parallelism to significantly boost computational efficiency as there are multiple operands.

The proposed 4-Bit design uses two Carry Save Adders (CSA-1 and CSA-2) which are discussed in the next section.

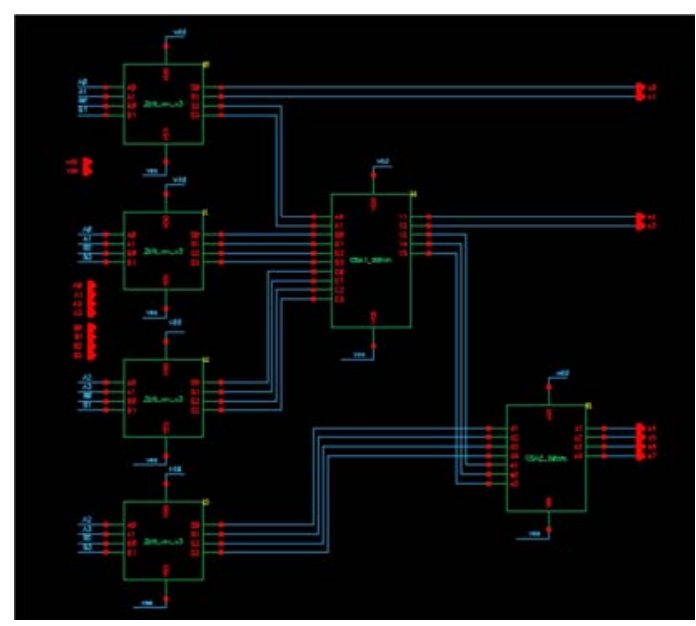

Figure 9. Schematic of the 4-Bit Vedic multiplier.

\subsubsection{Carry Save Adder (CSA-1)}

The design of CSA is modified to suit the needs of the 4- Bit multiplier. CSA-1 is used to add three numbers, two of which are four bits and the third in two bits wide. Figure 10 Shows the schematic of CSA-1 implemented in 90nm. CSA- 1 adds the partial products of the first three 2-Bit multipliers.

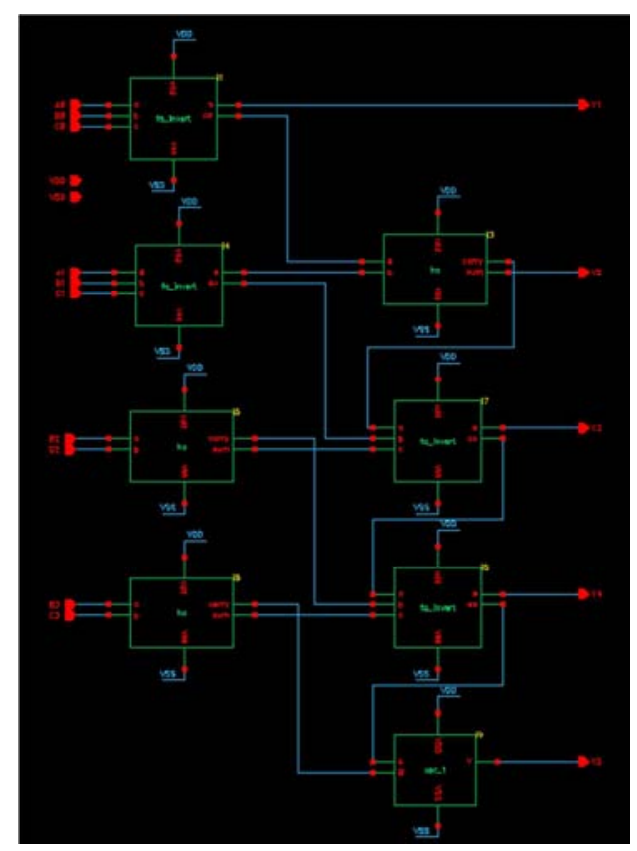

Figure 10. CSA-1 Schematic.

\subsubsection{Carry Save Adder 2 (CSA-2)}

Figure 11 shows the schematic of CSA-2. CSA-2 is modified to add two numbers, one of four bits that is the output of the fourth 2-Bit multiplier and the other of three bits that is the output of CSA-1.

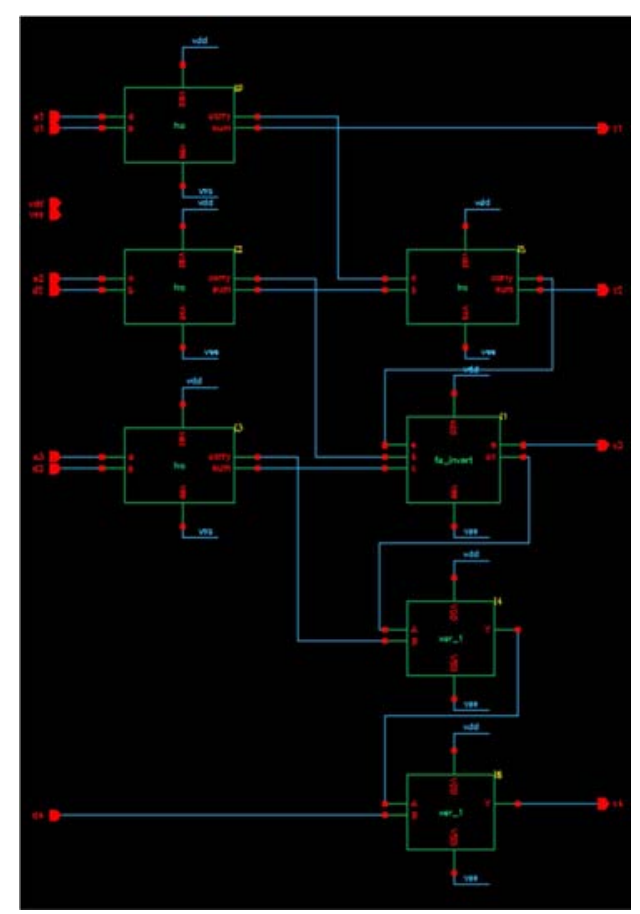

Figure 11. CSA-2 Schematic. 


\section{Layouts Designed in 90nm Technology}

The main goals of placement of cells are to achieve tim- ing, power, area optimization and least routing. To begin with, metal-2 tracks are laid horizontally uniformly spaced to achieve 7 track standard cells. Metal-3 tracks are laid out vertically as per the routing requirements. Placement of cells a crucial role in terms of area optimization, timing analy- sis and power consumption. The area occupied by the 2-Bit multiplier layout is $43.13 \mu \mathrm{m}^{2}$ and 4-Bit multiplier is $421.92 \mu \mathrm{m}^{2}$ in $90 \mathrm{~nm}$.

The substrate taps are placed within each cell and the metal wires run within the cell connecting the transistors properly. Supply rails are designed to ensure that they bear more current and have less resistance.

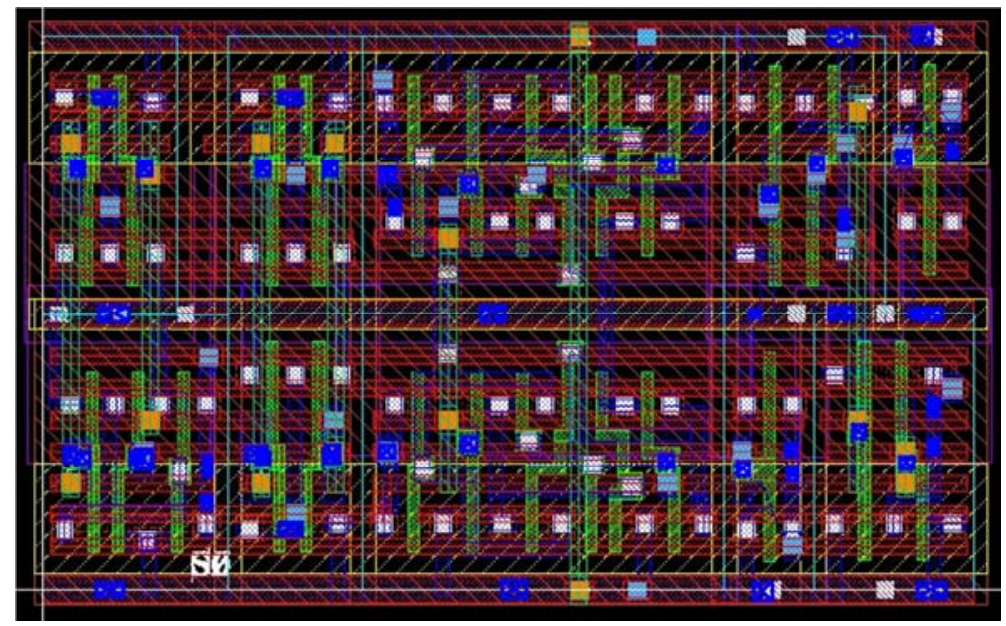

Figure 12. Layout design of the 2-Bitmultiplier.

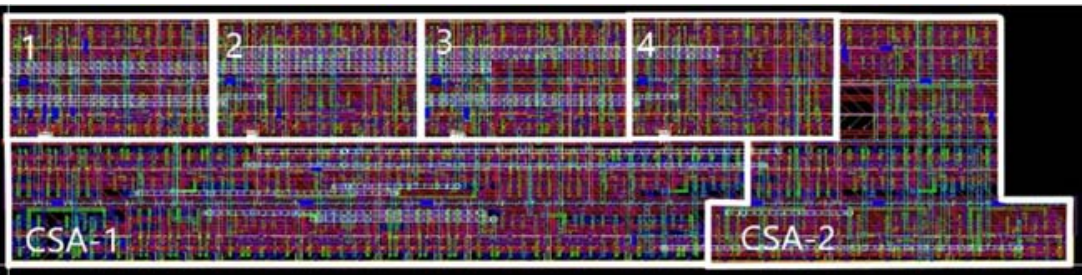

Figure 13. Layout design of the 4-Bit multiplier.

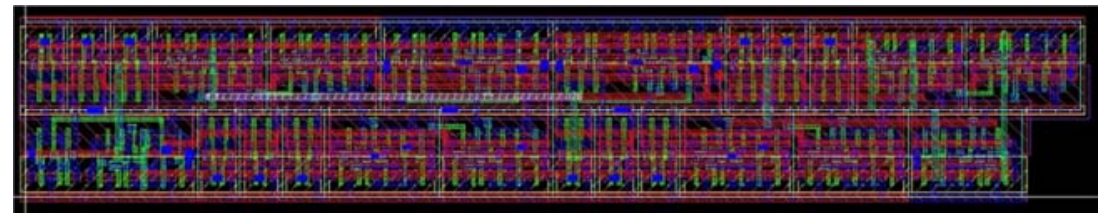

Figure 14. Layout design of CSA-1.

Inside each cell, the polysilicon runs vertically to form the transistor and the diffusion and the metall runs horizon- tally. Metal1 runs vertically where it does not interfere with other connections, it helps to save the area. The layouts de- signed are LVS and DRC clean to ensure high overall perfor- mance and reliability.

Figure 13 shows the 4-Bit multiplier layout which consists of four 2- Bit multipliers (labeled as 1, 2, 3 and 4), CSA-1 and CSA-2 as sub-blocks. Power rails run both horizontally and vertically at regular intervals and step down to metal-1 to power individual cells. Area occupied by CSA-1 and CSA-2 are $150.69 \mu \mathrm{m}^{2}$ and $93.47 \mu \mathrm{m}^{2}$ respectively.

Figure 15 shows the layout design of CSA-2. The cells are placed in this manner to have minimum global and local. routing congestion. VDD and VSS tracks run horizontally and adjacent cells share these tracks to minimize area occu- pied.

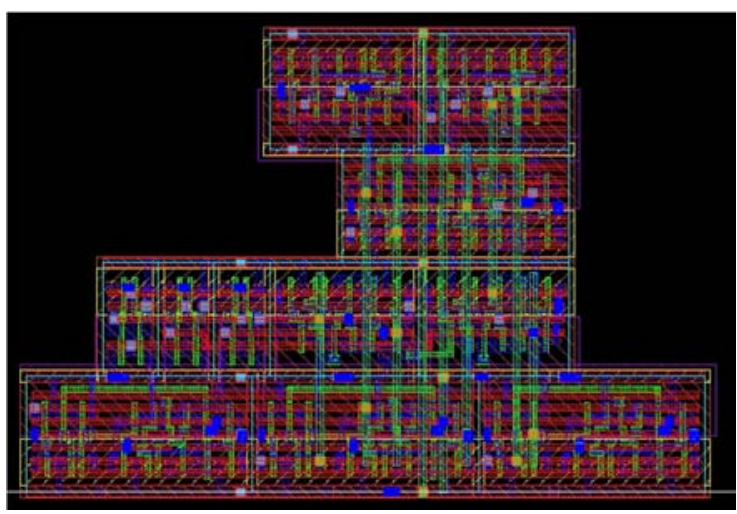

Figure 15. Layout design of CSA-2. 


\section{Results}

The performance analyses of the full adders are studied and results are shown in Table 1 . The proposed full adder saves $60 \%$ of power and is $20 \%$ faster the existing standard cell in $90 \mathrm{~nm}$ architecture in terms of power consumption, operating frequency and area savings, by reducing the transistor sizing according to fan-out required.

Table 1. Comparison of Full Adders.

\begin{tabular}{llll}
\hline Design analysis & & $\begin{array}{l}\text { Propagation Delay } \\
(\mathbf{p s})\end{array}$ & $\begin{array}{l}\text { Power } \\
(\mathbf{u W})\end{array}$ \\
\hline Standard FA $(90 \mathrm{~nm})$ & FA & 32.37 & 131 \\
Proposed FA $(90 \mathrm{~nm})$ & FA & 25.6 & 51.9 \\
\hline
\end{tabular}

Table 2. Comparison of 2-Bit multipliers.

\begin{tabular}{lll}
\hline Design analysis & $\begin{array}{l}\text { Propagation Delay } \\
(\mathbf{p s})\end{array}$ & $\begin{array}{l}\text { Power } \\
(\mathbf{u W})\end{array}$ \\
\hline Vedic 2-BitUT multiplier & 91 & 48 \\
Inverted Gate 2-Bit UT multiplier & 82.1 & 40.5 \\
\hline
\end{tabular}

The graphs shown in Figure 16 and Figure 17 depict the rise and fall delay time for the output MSB bit. The color red and blue indicate the propagation delay of the standard 4-Bit and the proposed 4-Bit multipliers respectively. One of the two 4 -Bit inputs is made to vary from $(0001)_{2}$ through $(1111)_{2}$, and the other is made constant $(1111)_{2}$.

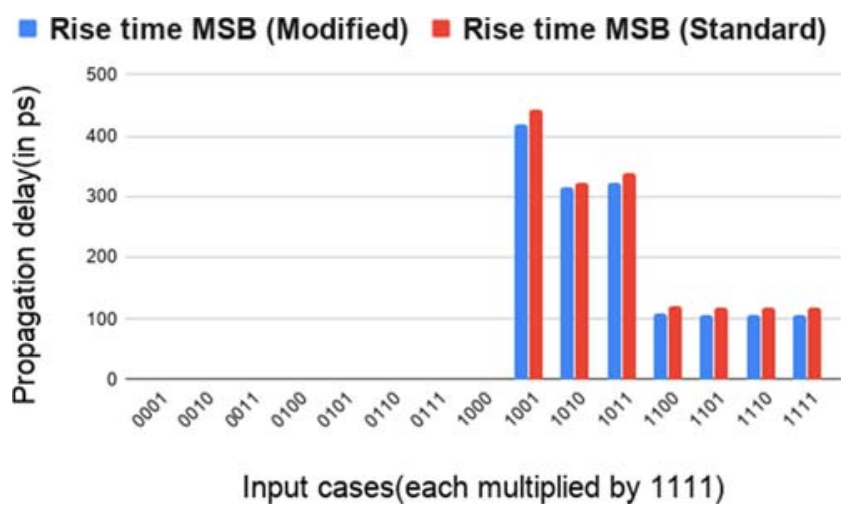

Figure 16. RISE delay comparison of 4-Bitmultipliers.

a Fall time MSB (Modified) = Fall time MSB (Standard)

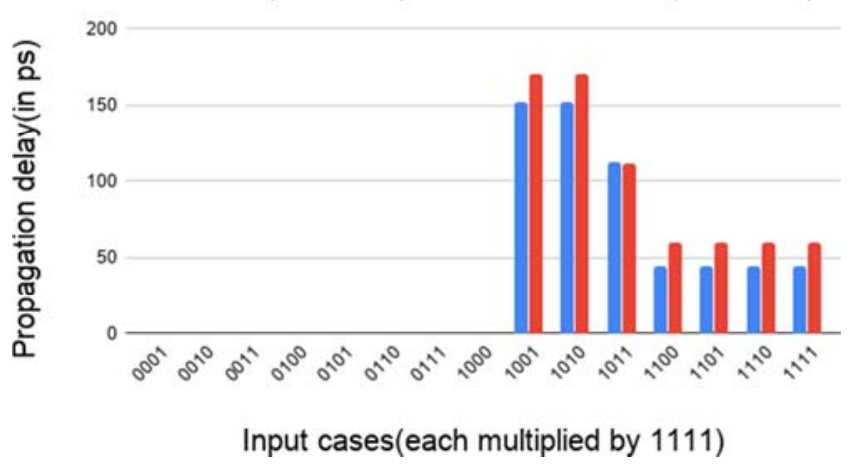

Figure 17. FALL delay comparison of 4-Bit multipliers

The performance analysis of the proposed 2-Bit multiplier is shown in Table 2, it is found to be faster and it consumes lesser power when compared to the existing design.

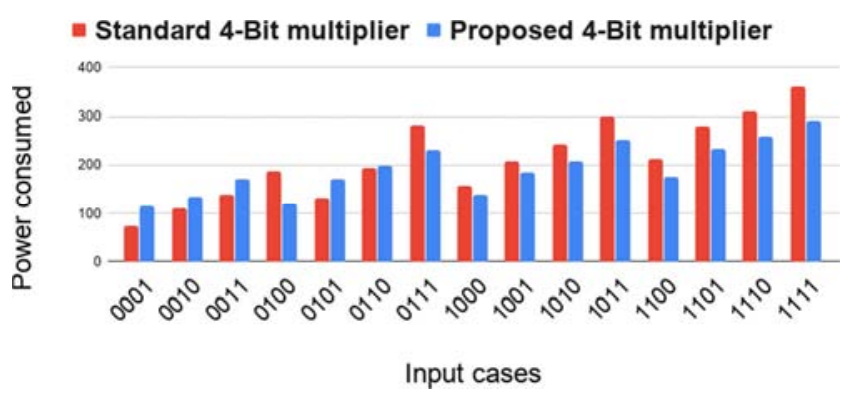

Figure 18. Power consumed comparision between the two designs.

The 4-Bit multiplier architecture is designed using both the 2-Bit designs and the results obtained favor the proposed Figure 18 depicts the power consumed by the standard 4- Bit multiplier designed using standard $90 \mathrm{~nm}$ cells and the power consumed by the proposed 4-Bit multiplier design. In the worst case, the standard design consumes $361.2 \mu \mathrm{W}$ and the proposed design consumes $290.2 \mu \mathrm{W}$, which reflects an $20 \%$ decrease in the power usage.

\section{Conclusion}

The traditional method of generating carry bit in the full adder makes use of AND and OR gates, which has been modified along with reducing the sizing of transistors without compromising on speed to create a faster and lower power consumption full adder. The standard full adder cell present in gpdk090 consumes $131 \mu \mathrm{W}$ of power in compari- son with $51.9 \mu \mathrm{W}$. This proposed full adder is implemented in the design of CSA's which improves the overall performance of the 4-Bit multiplier architecture.

The 2-Bit multiplier based on Urdhva Tiryagbhyam sutra is modified which enhances the performance in terms of operating frequency and power consumption. The 4-Bit multiplier is constructed in two variants, one using the standard full adder and 2-Bit Vedic multiplier design, the latter consisting of the proposed full adder and 2-Bit Vedic multiplier designs. The performance analyses of the two variants are conducted and the results obtained favor the proposed design.

\section{References}

[1] Jagadguru Swami Sri Bharati Krisna Tirthaji Maharaja. Vedic mathematics: Sixteen simple Mathematical For- mulae from the Vedas. Delhi 1965.

[2] Balraj Singh et al. "Analysis of CMOS based NAND and NOR Gates at $45 \mathrm{~nm}$ Technology". International Journal of Electronics, Electrical and Computational System (IJEECS), Vol. 6 (Issue 4), April 2017.

[3] Pawan Manoj Rathod et al. "Optimizing the Complex- ity of Matrix Multiplication Algorithm". Interna- tional Journal of Engineering Research \& Technology (IJERT), 5 (01), 2017. 
[4] MD. Belal Rashid et al. "VLSI Design and Implemen- tation of Binary Number Multiplier based on Urdhva Tiryagbhyam Sutra with reduced Delay and Area". In- ternational Journal of Engineering Research and Tech- nology., 2013.

[5] Harsha $\mathrm{R}$ et al. "Design of Vedic multiplier using Ur- dhva Tiryagbhyam Sutra". International Journal of Advance Research, Ideas and Innovations in Technol- ogy, 5 (3), 2019.

[6] Anuradha Singh and Neetu Gupta "Vedic mathematics For VLSI Design: A Review”. International Journal of Engineering Sciences \& Research Technology, 2017.

[7] Arish S and R. K. Sharma "An efficient binary mul- tiplier design for high speed applications using Karat- suba algorithm and Urdhva-Tiryagbhyam algorithm". Global Conference on Communication Technologies (GCCT), pages 192-196, 2015.

[8] N Janardan et al. "Implementation of 64 Bit Complex FloatingPoint Multiplier on FPGA using Vedic Math- ematics Sutra- Urdhva Tiryagbhyam". International Journal of Innovative Technology and Exploring Engi- neering (IJITEE), 9 (04), 2020.

[9] Chiranjit R Patel et al. "Vedic Multiplier in 45nm Technology". International Conference on Computing Methodologies and Communication (ICCMC), 2020.

[10] Chandrashekara M N and Rohith S "Design of 8 Bit Vedic
Multiplier Using Urdhva Tiryagbhyam Sutra With Modified Carry Save Adder". International Con- ference on Recent Trends on Electronics, Information, Communication \& Technology (RTEICT), 2019.

[11] Sandesh Sharma and Vangmayee Sharda "Design and Analysis of 8-bit Vedic Multiplier in 90nm Technology using GDI Technique". International Journal of Engi- neering \&Technology, 2018.

[12] Manikannan G et al. "Low Power High Speed Full Adder Cell with XOR/XNOR Logic Gates in 90nmTechnology". International Conference on Tech- nical Advancements in Computers and Communica- tions, 2017.

[13] Akshata R et al. "Analysis of Vedic Multipliers". Inter- national Conference on Computing, Communicationas and Energy Systems (ICCES), 2016.

[14] Neil H E Weste and David Money Harris. CMOS VLSI Design: A circuits and systems perpespective, 4 edi- tion. Pearson.

[15] Adel S Sedra and Kenneth C Smith. Microelectronics Circuits Theory and Applications.

[16] Performance Analysis of a Low-Power High-Speed Hybrid 1-bit Full Adder Circuit. In Partha Bhat- tacharyya et al., editors, IEEE Transactions on Very Large Scale Integration (VLSI) Systems, 2014. 